\title{
IMUNOGENECIDADE DE ACADÊMICOS DO CURSO DE ODONTOLOGIA EM RELAÇÃO À HEPATITE B
}

\section{ACADEMIC IMMUNOGENICITY OF COURSE OF DENTISTRY THROUGH HEPATITIS B}

\section{Sarah Freygang Mendes Pilati', Larissa Kobarg Cercal Patrianova², Alexssandra de Jesus Vitorett ${ }^{2}$}

\section{RESUMO}

Objetivo: determinar o perfil sorológico, através de marcadores da hepatite $B$ de acadêmicos do curso de odontologia. Metodologia: pesquisa descritiva com 70 graduandos do primeiro ao terceiro período do curso de odontologia no semestre de 2013/2, mediante a coleta de dados secundários, através da análise dos laudos sorológicos para os marcadores Anti-HBs; HBsAg; Anti-HBc Total. Resultados: um total de $87,14 \%$ dos acadêmicos já haviam realizado a vacina anteriormente à aplicação deste estudo, dos quais, apenas 18,6\% haviam realizado o exame Anti-HBs. A análise dos laudos evidenciou que, para $100 \%$ do grupo avaliado, os marcadores HBsAg e Anti-HBc Total foram não reagente e apenas 50,8\% obtiveram resultado reagente para o Anti-HBs. Conclusão: apesar da grande maioria dos acadêmicos já ter realizado a vacina contra hepatite $B$, apenas em uma parcela dos mesmos ocorreu soroconverção, sendo importante incentivar os acadêmicos a realizar o exame Anti-HBs, pois, este é fundamental para constatar a real presença da imunogenecidade.

Descritores: Hepatite B; Sorologia; Estudantes de Odontologia; Vacinação; Equipamentos de Proteção.

\section{ABSTRACT}

Objective: to evaluate the sorological profile of students of Dentistry through sorological markers of hepatitis $B$ and a knowledge of them in relation to Hepatitis $B$. The target population was 70 undergraduates from the first to the third period of the dentistry course in the year 2013/2. Methodology: this was an experimental study with application of a semi-structured questionnaire and blood test of Anti-HBs, HBsAg, Anti-HBc Total markers. Results: $87.14 \%$ of the students had held a vaccine previously implementation of this study. Only $18.6 \%$ of students had performed the Anti-HBs exam. The HBS-Ag and total HBC had the classification of non-reactive for all students, 31 (44.2\%) had a positive result for Anti-HBs, However, 39 (55.7\%) academic shad not positive result. Conclusion: although the vast majority of students have already done the hepatitis $B$ vaccine, only a portion of them occurred soroconvertion, it is important to encourage students to make the Anti-HBs test, because this is essential to establish the real presence of immunogenicity.

Descriptors: Hepatitis B; Serology; Dental Students; Immunization; Protective Devices.
${ }^{1}$ Mestre em Odontologia pela Universidade Federal de Santa Catarina (UFSC), Florianópolis, SC ,Brasil.

${ }^{2}$ Graduada em Odontologia pela Universidade do Vale do Itajaí, (UNIVALI), Florianópolis, SC, Brasil. 


\section{Introdução}

A hepatite é uma doença inflamatória que acomete o fígado e suas funções. Dentre as possíveis causas da hepatite, destaca-se a infecção pelo HBV (Vírus da Hepatite B) como a maior causadora de doenças hepáticas em todo o mundo ${ }^{1,2}$. Os trabalhadores da área de saúde, em geral, têm maior risco de infecção ${ }^{3}$. Entre os profissionais da área da saúde destacam-se os odontólogos ${ }^{4,5}$.

Segundo dados da Sociedade Brasileira de Infectologia (SBI) (2006), acredita-se que mais de 2 bilhões de pessoas no mundo estão infectadas pelo vírus da hepatite $B$ e que cerca de 360 milhões sofrem de infecção crônica por esse agente $^{6}$. Para o boletim epidemiológico de hepatites virais, em 2010 foram notifiาcados 13.188 casos de hepatite B no Brasil, sendo a maioria nas regiões Sudeste $37,7 \%$ e Sul $29,6 \% 7$.

As formas de infecção do HBV se dão por meio de relações sexuais desprotegidas, pois o vírus encontra-se no sêmen e secreções vaginais; realização de procedimentos sem esterilização adequada ou utilização de material descartável, tais como intervenções odontológicas e cirúrgicas, hemodiálise, tatuagens, perfurações de orelha, colocação de piercings; uso de drogas com compartilhamento de seringas, agulhas ou outros equipamentos; transfusão de sangue e derivados contaminados; transmissão vertical (mãe/filho); aleitamento materno; e acidentes perfurocortantes ${ }^{8}$.

Até o momento a melhor profilaxia no combate à infecção pelo vírus da hepatite $B$, é por meio da vacina, concomitante ao teste Anti-HBs, para avaliar a soroconversão da mesma, pois, a resposta vacinal depende de cada organismo e existe o risco de não se atingir níveis protetores de anticorpos, sendo necessário nestes casos doses de reforço ${ }^{9}$.

O HBV é altamente resistente, podendo sobreviver até sete dias no ambiente, com possibilidade de que quantidades minúsculas de sangue ou secreções contendo o vírus sejam capazes de transmitir a infecção através de diferentes formas, tais como, inalação de gotículas, aerossóis contaminados ou pelo transporte manual para a boca de partículas contaminadas presentes na superfície de balcões ${ }^{8,10}$.

Estima-se que o risco de contaminação com o vírus do HBV é de $6 \%$ a $30 \%$ após acidente com agulha ou materiais perfurocortantes ${ }^{5}$.

Dados da Unidade de Saúde Familiar e Comunitária - F7 (USFC- F7), situada na Universidade do Vale do Itajaí, até 13 de maio de 2013, a distribuição de acidentes com materiais perfurocortantes totalizaram 140 casos e destes $50 \%$ aconteceram no Curso de Odontologia. Como o curso de Odontologia é o curso que possui os maiores índices de acidentes com materiais perfurocortantes é imprescindível a realização do teste sorológico para confirmar que estes alunos estejam imunizados. Isso só poderia ser feito através do Anti-HBs que é um indicador de imunidade, por vacina ou por contato com o vírus, para confirmar com êxito a imunidade contra o VHB.

Baseado nessa evidências, objetivou-se avaliar se os alunos que não estiveram em contato clínico com pacientes atendidos dentro da universidade e que realizaram a vacina contra o vírus da Hepatite $B$ anteriormente a esta pesquisa, possuiam imunidade contra o vírus da Hepatite $B$, determinando assim o perfil sorológico dos acadêmicos do primeiro ao terceiro período de um curso de odontologia, em relação à hepatite $B$.

\section{Metodologia}

O estudo se caracterizou como uma investigação baseada nos princípios da pesquisa descritiva, mediante a coleta de dados secundários (laudos dos testes sorológicos) e primários (ficha de coleta de dados).

Foram critérios para a participação da pesquisa: a) estar regularmente matriculados em um dos três primeiros períodos do curso de odontologia da UNIVALI no segundo semestre de 2013; b) não ter realizado qualquer procedimento clínico na universidade, até a realização da coleta; e c) aceitar participar da pesquisa após assinar o termo de consentimento livre e esclarecido. Enquadraram-se nesses critérios um grupo composto por 70 acadêmicos.

Utilizou-se uma ficha de coleta de dados, contendo 02 questões de caracterização dos sujeitos e 02 utilizadas em complemento aos testes sorológicos. As duas etapas da pesquisa foram realizadas em sala de aula, sendo que primeiramente os acadêmicos preecheram as fichas, e posteriormente realizaram-se as coletas das amostras sanguíneas para a avaliação sorológica dos marcadores da hepatite B, sendo eles: o Anti-HBs, HBsAg, Anti-HBc Total. Ainterpretação dos resultados serviu para avaliação de imunidade em indivíduos sujeitos a risco de contágio com HBV e avaliação de eficácia do protocolo de imunização para HBV. 
Os procedimentos para a coleta do material foram baseados no Procedimento Operacional Padrão do Laboratório Municipal de Itajaí e foram realizadas em um período de 3 dias.

A coleta foi realizada pelo farmacêutico e bioquímico do laboratório de análises clínicas, em sala de aula. Foram geradas etiquetas de identificação das amostras sorológicas; uma etiqueta para cada tipo de material (Anti-HBs, HBsAg, Anti- HBc Total) e um comprovante de coleta.

Para coleta sanguínea, realizou-se a punção venosa na veia cubital mediana ou cefálica, utilizando agulha hipodérmica (22x7) e seringa descartável de $5 \mathrm{ml}$. Em seguida, o material foi colocado em um tubo sem anticoagulante, com gel separador para a preservação da amostra e transferência ao Laboratório Municipal para análise do material, dentro de caixas térmicas.

0 processo de análise ocorreu através de centrifugação durante 10 minutos a $3.500 \mathrm{rpm}$, pelo método de eletroquimioluminescência e quimioluminescência. O material (soro) foi armazenado em temperatura refrigerada de $5^{\circ} \mathrm{C}$. Os marcadores analisados foram Anti-HBs, HBsAg e Anti-HBc total. O marcador Anti-HBs (anticorpos contra o antígeno de superfície do HBV) é o único marcador que confere imunidade ao HBV, este, encontra-se no soro após a eficácia da vacinação contra HBV, podendo ser detectado em um período de trinta a noventa dias após a vacinação. Ou ainda, após o desaparecimento do HBsAg, sendo indicador de cura e imunidade. Foram considerados imunizados os que apresentaram Anti-HBs reagente ( $\geq 10 \mathrm{mUl} / \mathrm{ml}$ ). O HBsAg é o primeiro marcador sorológico detectável, surgindo uma a três semanas antes dos sintomas, seus valores de referência são: reagente $\geq 1,0$ e não reagente $<0,90$. Já, o Anti-HBc total é o marcador utilizado na triagem para a hepatite B por detectar tanto o anticorpo lgG quanto o anticorpo $\lg M$. Sendo assim, ao receber como resultado o Anti-HBc total reagente, é importante definir se o resultado advém dos altos títulos de lgG (imunidade por infecção passada ou imunidade por resposta vacinal) ou pelos altos títulos de lgM (fase aguda). Seus valores de referência são: ausência de anticorpo (não reagente) e presença de anticorpo (reagente). Após a análise e interpretação dos resultados, foi realizado devolutiva aos participantes da pesquisa.

As variáveis estudadas foram: a relação dos resultados do teste sorológico com a imunidade dos alunos participantes e suas respostas ao questionário.

Os dados obtidos nas duas etapas foram tabulados utilizando o programa Excel 2010, e posteriormente calculada a frequência relativa para cada item analisado. Utilizou-se como método de estatística o teste Qui-quadrado com correção de Yates.

O estudo respeitou os princípios éticos de pesquisa envolvendo seres humanos constantes da Resolução CNS no 196, do Conselho Nacional de Saúde ${ }^{11}$. Os acadêmicos participantes não foram identificados, sendo o sigilo e a privacidade das informações assegurados. As pessoas que participaram da pesquisa assinaram um 'Termo de Consentimento Livre e Esclarecido. Esta pesquisa teve início após a aprovação do mesmo no Comitê de Ética da UNIVALI, sob parecer 447.192.

\section{Resultados}

Através da análise de resultados obtidos por meio da realização dos exames , foi possível constatar e traçar o perfil sorológico dos acadêmicos de odontologia da UNIVALI. Participaram da pesquisa 70 alunos voluntários, sendo 19 do primeiro período, 28 do segundo período e 23 do terceiro período. Com relação ao gênero, $57(81,4 \%)$ eram do gênero feminino e $13(18,6 \%)$ masculino. Em relação à realização da vacina contra o HBV, identificou-se que, 61 (87,14\%) haviam realizado a mesma em algum momento anterior à esta pesquisa. Na tabela II, encontra-se a frequência de vacinados, segundo o período de matrícula. Nota-se que o número de acadêmicos sem vacina foi significativamente inferior quando comparados aos acadêmicos com vacina ao considerarmos o teste Qui-quadrado com correção de Yates $\left(X^{2}=6,279 ; p=0.0047\right)$.

Tabela I - Frequência de respostas sobre a realização da vacina contra o HBV, segundo o período de matrícula dos sujeitos da pesquisa.

\begin{tabular}{|c|c|c|c|c|c|}
\hline \multirow{2}{*}{ Semestre } & Sim & \multicolumn{2}{|c|}{ Não } & \multicolumn{2}{|c|}{ Total } \\
\hline & N \% & & $\%$ & & $\%$ \\
\hline Primeiro & 1789,5 & $\mathrm{O} 2$ & 10,5 & 19 & 100 \\
\hline Segundo & 2589,3 & $\mathrm{O3}$ & 10,7 & 28 & 100 \\
\hline Terceiro & 1982,6 & 04 & 17,4 & 23 & 100 \\
\hline
\end{tabular}

Fonte: Dados da pesquisa 
Quanto à realização do teste Anti-HBs anteriormente a esta pesquisa, apenas $13(18,6 \%)$ dos analisados haviam realizado o exame (Tabela II).

Tabela II- Frequência de pesquisados que realizaram o teste Anti-HBs, por período de matrícula

\begin{tabular}{c|cc|cc|ccc}
\hline \multirow{2}{*}{ Semestre } & \multicolumn{2}{|c|}{ Sim } & \multicolumn{2}{|c|}{ Não } & \multicolumn{2}{|c}{ Total } \\
\cline { 2 - 8 } & \multicolumn{2}{|c|}{$\mathbf{N}$} & $\%$ & \multicolumn{2}{|c|}{$\mathbf{N}$} & $\%$ & \multicolumn{2}{|c}{$\mathbf{N}$} & $\%$ \\
\hline Primeiro & 0 & 0,0 & 19 & 100,0 & 19 & 100 \\
\hline Segundo & 03 & 10,7 & 25 & 89,3 & 28 & 100 \\
\hline Terceiro & 10 & 43,5 & 13 & 56,5 & 23 & 100 \\
\hline
\end{tabular}

Fonte: Dados da Pesquisa.

Verificou-se junto aos laudos laboratoriais que dos 61 acadêmicos que já haviam realizado a vacina, apenas $31(50,8 \%)$ obtiveram resultado reagente ( $\geq 10 \mathrm{UI} / \mathrm{ml})$ (Tabela III).

Tabela III- Resultados do teste de soroconversão (Anti-HBs), obtido através da análise sanguínea dos acadêmicos dos três períodos

\begin{tabular}{c|rc|cc|cc|}
\hline \multirow{2}{*}{ Semestre } & \multicolumn{2}{|c|}{ Reagente } & \multicolumn{2}{|c|}{ Não Reagente } & \multicolumn{2}{|c}{ Total } \\
\cline { 2 - 8 } & $\mathbf{N}$ & $\%$ & $\mathbf{N}$ & $\%$ & $\mathbf{N}$ & $\%$ \\
\hline Primeiro & 12 & 70,5 & 07 & 41 & 17 & 100 \\
\hline Segundo & 10 & 40 & 18 & 72 & 25 & 100 \\
\hline Terceiro & 09 & 47,4 & 14 & 73,6 & 19 & 100 \\
\hline
\end{tabular}

Fonte: Dados da Pesquisa.

É importante frisar que nenhum acadêmico obteve resultado reagente para os marcadores HBsAg e anti-HBc Total.

\section{Discussão}

Sabe-se que a hepatite B merece um enfoque especial, por apresentar risco ocupacional reconhecido, sendo recomendada a vacinação a todos os profissionais e estudantes de odontologia, prevenindo infecções antes que ocorra a exposição. Diversos estudos confirmam a maior suscetibilidade para profissionais da saúde, por estarem constantemente expostos a fluidos e secreções, sendo assim, aumentam os riscos de contaminação 2, 9,12,13,14,15,16,17,19,20,21.

$O$ principal meio de prevenção contra o vírus da hepatite $B(\mathrm{HBV})$ se dá através da imunização completa, sendo necessário as três doses, no período de 0; 30; 180 dias, afim de estimular produção de anticorpos acima de $10 \mathrm{UI} / \mathrm{ml}$. Portanto, a proteção efetiva de profissionais e pacientes, a adoção de protocolos e de condutas de biossegurança devem ser rigorosamente seguidos $5,12,16,17,19,20,21,22,23,24,25,26$.

O que se preconiza, é que indivíduos vacinados realizem o teste anti-HBs 30 dias após o esquema completo de vacinação (03 doses), para comprovação de efetividade das mesmas. Aqueles com anti-HBs não reagente, após o segundo esquema completo com três doses, devem ser considerados não respondedores e suscetíveis, sendo que esta não resposta também pode estar ligada a fatores genéticos e HLA específicos devendo ser encaminhados para avaliação médica ${ }^{17}$.

Através de estudos sobre as características dos pacientes, podemos entender a associação entre os antigénios dos leucócitos humanos (HLA) e a susceptibilidade a infecções, tais como pelo HBV. A susceptibilidade à persistência ou resolução da infecção por HBV depende da resposta imune e pode ser explicada por factores imunogenéticos no hospedeiro, e há evidência de que em certos grupos, tais como homens e em indivíduos com menos de 39 anos, foi associada com o HLA-DRB1 * 09 e DRB1 ${ }^{*} 08$ alelos, respectivamente ${ }^{18}$. 
O bloqueio epidemiológico da transmissão de infecções e proteção dos profissionais tem como grande aliado à vacinação, a maioria disponível através do Sistema Único de Saúde, que reduz o risco de infecções. A imunização durante 0 treinamento/aprendizagem (em especial nas universidades) deve ser incentivada com intuito de prevenir a infecção antes que ocorra qualquer tipo de exposiçãa $0^{5,24,13}$.

É importante também, ressaltar a verificação da eficácia da imunização contra hepatite B com o exame Anti-HBs, sendo que este, deve ser realizado num período de pelo menos 30 dias após a terceira dose da vacina. O Anti-HBs é um exame importante que detecta presença de anticorpos para a doença, dando uma segurança necessária ao profissional, já que a hepatite $B$ é a doença de maior prevalência em profissionais que exercem atividade de risco, sobretudo na área odontológica ${ }^{1314,19,25}$. É válido ressaltar ainda, que a imunização se faz importante tanto para o profissional, quanto para 0 paciente, pois, durante um atendimento odontológico a contaminação pelo HBV pode ocorrer do paciente para o dentista, do dentista para o paciente e de um paciente para o outro ${ }^{26}$.

Assim, a escolha por esta pesquisa com acadêmicos que ainda não iniciaram as práticas clínicas na universidade foi com intuito de ação preventiva. $O$ estudo pode ser considerado como um mecanismo de alerta, possibilitando 0 encaminhamento dos alunos para a efetivação do esquema vacinal, concomitante ao teste de soroconversão. E assim procedendo-se, tem-se a garantia de que, quando estes acadêmicos iniciarem as atividades clínicas, estarão com esquema vacinal completo, sendo mais uma barreira de proteção contra essa infecção, sendo ainda, que nos períodos iniciais os graduandos possuem menos destreza manual, estando exposto assim a maior risco.

Um dos principais questionamentos desta pesquisa foi baseado na realização da vacina contra a hepatite $B$ em alguma fase da vida. Percebeu-se que a grande maioria já havia realizado a vacina anteriormente à aplicação deste estudo. Este resultado corrobora com outras pesquisas ${ }^{26}$.

A principal medida de prevenção da hepatite $B$ é representada pelas vacinas, e sua resposta máxima à imunização ocorre cerca de seis semanas após a terceira dose, existindo correlação direta desses títulos com a persistência do AntiHBs após a imunização ${ }^{24,25}$.

A análise do perfil sorológico dos acadêmicos que integraram este estudo evidenciou que a maioria nunca havia realizado o teste (Anti-HBs). Esta condição, também, foi relatada em outros estudos ${ }^{26,27}$. A soroconversão após a vacinação contra o vírus da hepatite $B$ entre profissionais da área da saúde é essencial, e estes devem ser incentivados a completarem o esquema de vacinal contra a hepatite $\mathrm{B}$, pois a proteção da vacina aumenta com o número de doses administradas ${ }^{25}$.

Considera-se de extrema importância a realização da dosagem de Anti-HBs após a vacinação, visto que a resposta à vacina depende de cada organismo e existe o risco de não se atingir níveis protetores de anticorpos, sendo necessárias doses de reforço $0^{9}$. Na literatura revisada, foram identificados vários estudos sobre a determinação da prevalência de marcadores sorológicos parahepatiteB, comoHBsAg; Anti-HBseAnti-HBcTotal, comprovandoa importância deste procedimento 4,5,12,1420,22,25,28.

A partir dos laudos emitidos para os testes sorológicos, referentes aos três marcadores da hepatite $B$, identificouse que os marcadores HBsAg e anti-HBc Total foram não reagentes para todos os acadêmicos, significando, assim, que nenhum dos acadêmicos apresentou contato com o vírus da hepatite $B$, e que também não houve no soro, a presença de anticorpos IgG e IgM, respectivamente. $O$ anticorpo lgG é conhecido como anticorpo de memória, e tipicamente persiste por toda vida. Já o anticorpo lgM é aquele presente em infecções agudas ou recentes. Estudos publicados mostram resultados semelhantes aos nossos, evidenciando abaixa prevalência destes marcadores em suas populações $20,22,25,28$. A dificuldade de encontrar estudos semelhantes ao nosso em relação à população estudada e/ou aos resultados apresentados de maneira separada para cada marcador foi desafiador, pois, pouco há na literatura pesquisas que apresentam resultados separados para os marcadores, dificultando a interpretação de seus estudos ${ }^{4,22}$.

Na população estudada, a eficácia da vacina contra a hepatite $B$ foi comprovada em trinta e um (31) acadêmicos que obtiveram resultado reagente para Anti-HBs ( $\geq 10,0 \mathrm{mUl} / \mathrm{ml})$, tendo em vista que não houve contato com HBV em nenhum dos casos, ou seja, a imunidade foi adquirida através da vacina. Todavia, em trinta (30) acadêmicos que alegaram ter recebido a vacina, a soroconversão não aconteceu, pois obtiveram títulos $<10,0 \mathrm{mUl} / \mathrm{ml}$, mostrando que o fato de ter realizado a vacina não significa seguramente que estarão imunes, frente a infecção pelo HBV.

Para a Sociedade Brasileira de Pediatria, os fatores que podem afetar a resposta à vacina incluem: modo de conservação da vacina, local da aplicação, sexo, idade, peso maior que $70 \mathrm{~kg}$, obesidade, fumo, fatores genéticos, doenças crônicas e condição nutricional e imunológica ${ }^{29}$. Após a vacinação a soroconversão pode diminuir frente aos problemas de insuficiência renal, diabetes, doença hepática crônica, infecção por vírus da imunodeficiência humana, tabagismo e obesidade ${ }^{5}$. Todavia, não podemos afirmar que a população investigada nesta pesquisa possa ser incluída nestes grupos. 
Diversos estudos onde se analisou a soroconversão (Anti-HBs) após o esquema vacinal completo, nos mostram que a imunização não alcançou os níveis absolutos, ou seja, mesmo após a vacinação contra hepatite $B$, os valores para Anti-HBs são inferiores a $10 \mathrm{UI} / \mathrm{ml}$, indicando que, mesmo após vacinação, nem todo profissional adquire número de anticorpos suficientes contra o HBV. No entanto, eventualmente, o anti-HBc pode ser 0 único indicador da imunidade natural detectável sorologicamente, pois com o tempo o nível de anti-HBs pode tornar-se indetectável,14,22,26.

Após a análise dos laudos sorológicos os mesmos foram entregues a cada aluno individualmente, tendo em vista que os acadêmicos que apresentaram resultados incompatíveis com imunidade para o vírus da hepatite $B$ (índices menores que $10 \mathrm{mUl} / \mathrm{ml}$ ) foram orientados a procurar a sala de vacinação na Unidade de Saúde situada dentro da universidade ou a Unidade de Saúde mais próxima da sua residência, afim de realizarem a vacinação seguida do teste para comprovação da mesma.

Diversos estudos mostraram que há uma forte necessidade de implementação de campanhas de conscientização que reforcem a importância da imunização concomitante a verificação de soroconversão da mesma 2,15,26,30.

Por fim, sugeriu-se a implementação de um protocolo para comprovação de vacinação juntamente com o teste de soroconversão, dentro do curso de odontologia da Universidade do Vale do Itajaí, a fim de promover um real conhecimento do acadêmico em relação à sua imunidade, e desta forma conseguir promover saúde e segurança destes acadêmicos, que em um futuro muito próximo, estarão atuando nas clínicas de odontologia dentro da universidade e unidades básicas de saúde da região.

\section{Considerações Finais}

Em relação aos resultados obtidos, a grande maioria dos acadêmicos já havia realizado a vacina contra hepatite $B$, todavia, constatou-se que apenas em uma parcela dos alunos ocorreu soroconverção. Tal fato nos mostra a necessidade de implementação de estratégias específicas para conscientizar os acadêmicos da saúde que além da importância da vacinação, a significância do teste de soroconversão, pois, este é fundamental para constatar a real presença da imunidade, sendo esta a maneira mais eficaz de prevenção, tendo em vista que apenas a apresentação da carteira da vacinação não significa o êxito no esquema vacinal contra HBV.

\section{Referências}

1. Castelo A, Pessôa MG, Barreto TCBB, Alves MRD, Araújo DV. Estimativas de custo de Hepatite crônica B no Sistema Único de Saúde brasileiro em 2005. AMB rev. Assoc. Med. Bras. 2007; 20 (6): 486-489.

2. Freitas DA, Maurício CC, Santos ALD, Caballero AD, Hernandez, Pereira MM. Conhecimento de acadêmicos de odontologia sobre Hepatite B. Rev. bras. cir. cabeça pescoço. 2011; 40(1):30-33.

3. Jardim EMA, CarvalhoPAM, Silva RP. Vacinação contra Hepatite $B$ e resposta vacinal em trabalhadores da área da saúde envolvidos em acidentes com material biológico. Acta de Ciências \& Saúde. 2013; 2 (2): 14-24.

4. FernandesJV, Braz RFS, Francisco Neto FV, Silva MA, Costa NF, Ferreira AM. Prevalência de marcadores sorológicos do vírus da hepatite B em trabalhadores do serviço hospitalar. Rev. saúde pública. 1999; 33(2): 122-128.

5. Moreira MG, Evangelista PF, Athayde LA. Perfil sorológico dos marcadores de Hepatite B em profissionais e acadêmicos da área da saúde. Rev. bras. Clin. 2010;42(4):255-259.

6. Sociedade Brasileira de Infectologia .The Brazilian Journal Of Infectious Diseases: I Consenso da Sociedade Brasileira de Infectologia para o diagnóstico e manuseio da hepatite B (e Delta). Salvador. 2006; 10 (1).

7. Ministério da Saúde (BR) Secretaria de Vigilância em Saúde. Secretaria de Vigilância em Saúde Departamento de DST, Aids e Hepatites Virais. Boletim epidemiológico hepatites virais. Brasília, Ministério da Saúde, 2012. 168 p.

8. Ministério da Saúde (BR) Secretaria de Vigilância em Saúde. Departamento de Vigilância Epidemiológica. A, B, C, D, E de Hepatites virais para comunicadores. Brasília, Ministério da Saúde, 2005. 24 p.

9. Domingues $\mathrm{BD}$,Cota $\mathrm{GS}$, Silva RMM. Avaliação da resposta imunológica à vacinação para hepatite $\mathrm{B}$, em profissionais de laboratórios de analises clínicas no município de Timóteo / MG. Farmácia \& Ciência. 2010 Ago-Dez,1:41-51.

10. Ministério da Saúde (BR) Secretaria de Vigilância em Saúde. Departamento de Vigilância Epidemiológica. Hepatites virais, o Brasil está atento. Brasília: Ministério da Saúde, 2008. 42 p 
11. Conselho Nacional de Saúde. Comissão Nacional de Ética e Pesquisa. Resolução nº 196, 10 de outubro de 1996, que regulamenta as pesquisas envolvendo seres humanos. Ministério da Saúde; Diário Oficial da União, Brasília, p. 1; 16 outubro de 1996. Seção 1.

12. Moreira RC, Saraceni CP, Oba IT, Spina AM, Pinho JRR, Souza LTM, Omoto TM, Kitamura C, Oselka G. Soroprevalência da hepatite $\mathrm{B}$ e avaliação da resposta imunológica à vacinação contra a hepatite $\mathrm{B}$ por via intramuscular e intradérmica em profissionais de um laboratório de saúde pública. J. bras. patol. med. lab. Rio de Janeiro. 2007 Out;43(5):313-318.

13. Assis CO. Caminho da Odontologia Contemporânea. Rev. bras. odontol. 2008 Jan.-Jun;65(1):72-75.

14. Storer FL. Soropositividade, cobertura e resposta vacinal para hepatite viral do tipo B em cirurgiões dentistas em Porto Velho, Rondônia, Brasil. 2008. 66f.

Dissertação (mestrado) Departamento de Odontologia, Universidade de Taubaté.

15. Vieira SC, Silveira DMR, Cardoso VC, Pinheiro JJV, Araújo EC. Cobertura vacinal para o vírus da hepatite B em cirurgiões dentistas. Rev. para. med. 2008 Out-Dez; 22 (4):1-4.

16. Silva FAG, Miasato JM. Hepatites virais: um fator de risco na prática odontológica. Rev. bras. odontol. 2009 JanJun;66(1):23-27.

17. Ministério da Saúde (BR). Secretaria de Vigilância em Saúde Departamento de DST, Aids e Hepatites Virais. Manual A B C D E das Hepatites Virais para Cirurgiões Dentistas. Brasília, Ministério da Saúde, 2010. 100 p.

18. Corrêa B, Lopes EPA, Albuquerque MFPM, Dourado L. Association between HLA-DRB1* polymorphisms and hepatitis B infection in a Brazilian population. Revista Associação Brasileira de Medicina. 2012; 58 (5): 537-542.

19. Milani RM, Canini SRMS, Garbin LM, Teles AS, Gir E, Pimenta FR. Imunização contra hepatite B em profissionais e estudantes da área da saúde: revisão integrativa. Rev. eletrônica enferm. 2011 Abr-jun; 13(2): 323-330.

20. Oliveira CMA, Nunes MRT, Nunes HM, Soares MCP. Prevalência de marcadores sorológicos do vírus da hepatite $B$ em profissionais de saúde de um laboratório de pesquisa na Amazônia oriental, Estado do Pará, Brasil, 2007 a 2009. Epidemiol. 2012 Out-Dez; 21(4):609-616.

21. Ganczak MA. Cross-Sectional Study on Anti Hepatitis B Immune Status in Vaccinate Healthcare Workers in the West Pomeranian Region of Poland. Hepat Mon. 2012; 12(3):185-189.

22. Carneiro AF, Daher RR. Soroprevalência do Vírus de Hepatite B em anestesiologistas. bras. anestesiol. 2003; 53(5):672-679.

23. Antunes H, Macedo M, Estrada A. Taxa de cobertura vacinal com imunização para o vírus da hepatite B. Acta med. Port. 2004;17:303-308.

24. Petry A, Kupek EJ. Efetividade das vacinas anti-VHB (DNA-recombinante) em doadores de sangue de uma região endêmica para hepatite B no sul do Brasil. Rev. Soc. Bras. Med. Trop. 2006 Set-Out; 39(5):462-466.

25. Vireira TB, Pereira R, Santos KF, Leal DBR. Soroconversão após a vacinação para hepatite $B$ em acadêmicos da área da saúde. Revista Saúde (Santa Maria). 2006;7(1):13-21.

26. Aangelo AR, Queiroga AS, Gonçalves LFF, Santos SD, Souza CDS, Soares MSM. Hepatite B: Conhecimento e Prática dos Alunos de Odontologia da UFPB. Pesqui. bras. odontopediatria clín. integr. 2007 Set-Dez;7(3):211-216.

27. Pinheiro J, Zeitoune RCG. Hepatite b: conhecimento e medidas de biossegurança e a saúde do trabalhador de enfermagem. Esc. Anna. Rev. Enferm (online). 2008 Jun; 12(2): 258-264.

28. Tonial GC,Passos AM, Livramento A, Scaravelli NG, BatschauerAPB, Bueno EC, etal. Hepatitis B marker seroprevalence and vaccination coverage in adolescents in the city of Itajaí, State of Santa Catarina, southern Brazil, in 2008. Rev. Soc. Bras. Med. Trop. 2011 Jul- Ago; 44 (4): 416-419.

29. Scaramuzzi DR. Projeto diretrizes: Vacina Contra Hepatite B [internet]. 2002. Disponível em: <http://www. projetodiretrizes.org.br/projeto_diretrizes/115.pdf>.

30. Buneo M, Matijasevich A. Avaliação da cobertura vacinal contra hepatite B nos menores de 20 anos em municípios do Estado do Rio Grande do Sul, Brasil. Epidemiol. serv. saúde. Brasília, 2011 Jul-Set; 20(3):345-354. 


\section{Sarah Freygang Mendes Pilati}

Endereço para correspondência - Rua: Uruguai, $n^{\circ} 458$,

Setor C5 - Térreo - Sala 106, Campus Itajaí

Bairro: Centro, CEP: 88302-202, Itajaí, SC, Brasil.

E-mail: sarahfreygang@gmail.com

Lattes: http://lattes.cnpq.br/2393055412472410

Larissa Kobarg Cercal Patrianova - lara_kcp@hotmail.com

Alexssandra de Jesus Vitoretti - sandravitoretti@gmail.com

\section{Enviado em 01 de outubro de 2015.} Aceito em 01 de abril de 2016. 